\title{
Nursing and Health Care
}

\section{Lipoid Pneumonia Due to Aspiration of Oil Products: Two Case Reports} Gokhan Perincek $^{1}$, Sema Avci $^{2^{*}}$ and Emrah Batmaz ${ }^{3}$

\section{Affiliation}

${ }^{1}$ Respiratory Medicine, Kars Harakani State Hospital, Turkey

${ }^{2}$ Emergency Medicine, Amasya University, Sabuncuoglu Serefeddin Research and Training Hospital, Turkey

${ }^{3}$ Respiratory Medicine, Tekirdag State Hospital, Tekirdag, Turkey

*Corresponding author: Sema Avci, Emergency Medicine, Amasya University, Sabuncuoglu Serefeddin Research and Training Hospital, Turkey, E-mail: dnzlsema@gmail.com

Citation: Perincek G, Avci S and Batmaz E. Lipoid pneumonia due to aspiration of oil products: Two case reports (2018) Nursing and Health Care 3: 69-70

Received: Oct 10, 2018

Accepted: Oct 15, 2018

Published: Oct 22, 2018

Copyright: @ 2018 Perincek G, et al., This is an open-access article distributed under the terms of the Creative Commons Attribution License, which permits unrestricted use, distribution, and reproduction in any medium, provided the original author and source are credited.

\begin{abstract}
Keywords: Lipoid pneumonia, Aspiration, Oil products

\section{Introduction}

Lipoid pneumonia which is an uncommon disease, with a frequency of $1-2.5 \%$ caused by the presence of lipid in the alveoli [1,2]. Lipoid pneumonia is classified into two major groups; exogenous and endogenous/idiopathic pneumonia [2]. Acute exogenous pneumonia is typically caused by aspiration of a sum of petroleum-based products [2]. It is typical for children due to accidental poisoning [2]. Chronic exogenous pneumonia commonly results from recurrent episodes of animal fat, mineral or vegetable oils [2]. Exogenous pneumonia is typically for older patients but sometimes it can be seen in children suffering from aspiration [2]. Lipoid pneumonia commonly presents with subtle onset and respiratory symptoms such as cough, dyspnea, chest pain, fever, hemoptysis, systemic findings and radiological findings such as pulmonary opacities, consolidations, pneumomediastinum, pneumothorax, pleural effusions, pneumotoceles and nodules [1,2]. We aim to present here two cases of exogenous pneumonia and their radiological findings.
\end{abstract}

Lipoid pneumonia which is a rare disorder, results from accumulation of lipids in the alveoli. Lipoid pneumonia has two types; exogenous pneumonia which results from deposition of inhaled oil in the lung and endogenous pneumonia which results from breakdown of lung distal to obstructed airway. Tumor or bronchiolitis obliterans or following chemotherapy or radiotherapy lead to release of cholesterol and other lipids in alveoli. We report clinical and radiological findings of two cases with exogenous lipoid pneumonia due to aspiration of oil products.

\section{Case Report 1}

A 16-year-old boy admitted to emergency room (ER) with nausea, emesis, cough, sputum and chest pain. His medical history and family history were unremarkable. He said that he took diesel oil with a hose by mouth from the chemical reservoir and he aspirated it accidently one day before. The patients general condition was worsened during the examination and he was intubated in ER. In the respiratory system examination; inspiratory rales were heard more prominent in the on the right lung basal side. Figure 1a-1c show chest radiographs of him on the admission, 10th day and 30th day, respectively. The patient was given antibiotics (Meropenem 1 gram, three times a day) in the intensive care unit. The patient was extubated and taken to Respiratory medicine service on the 4th day of the treatment. Verbal consent was obtained from the patient for this case presentation.

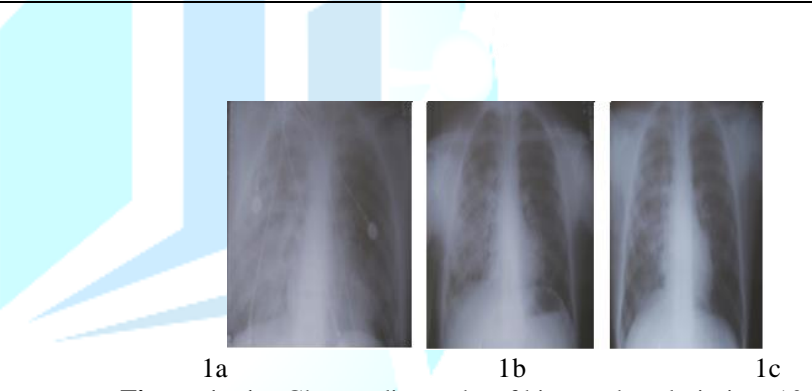

Figure 1a-1c: Chest radiographs of him on the admission, 10th day and 30th day, respectively.

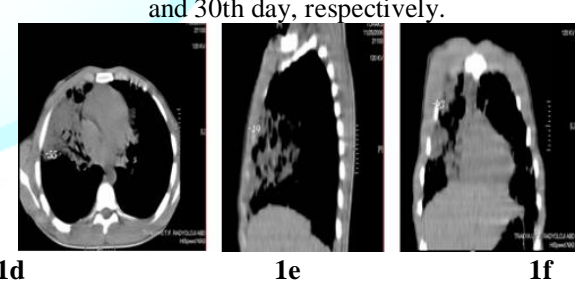

Figure 1d-1f: Chest computed tomography findings of patient.

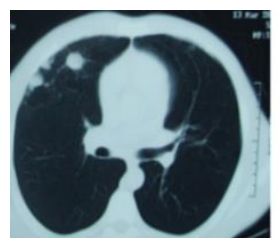

$1 \mathrm{~g}$

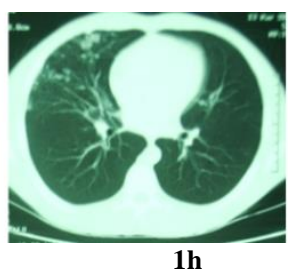

Figure $1 \mathrm{~g}$ and $1 \mathrm{~h}$ : Noduler sequela was seen on the patients chest tomography in the sixth month, after discharge.

Citation: Perincek G, Avci S and Batmaz E. Lipoid pneumonia due to aspiration of oil products: Two case reports (2018) Nursing and Health Care 3: 69- 70 
Chest computed tomography showed parenchymal consolidation areas containing air bronchograms at fat density. Density measurements were made in vertical, axial and sagittal sections. Figure 1d-1f show chest computed tomography findings of exogenous pneumonia for this patient. Antibiotherapy of the patient continued with ampicillinsulbactam $(4 * 1.5$ gram intravenous for a day) and completed to 15 days. The patient with clinical and radiological improvement was discharged at the end of 15 days. After discharge, nodular sequela was seen on the patients chest tomography (Figure 1g and 1h).

\section{Case Report 2}

A 72-year-old man admitted to emergency room with cough, high fever and hemoptysis. The patient accidentally drank a glass of gas oil five days before. The patient had only hypertension disease. 4 hours after the incident, the patient was admitted to a hospital and treated with antibiotics

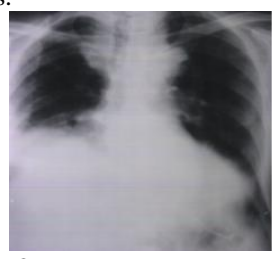

$2 \mathrm{a}$

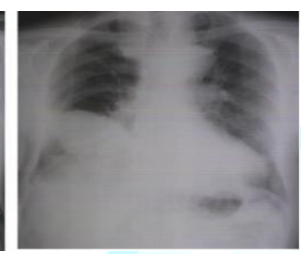

$2 \mathrm{~b}$
Figure 2a and 2b: Chest radiographs of the patients admission to hospital and hospital discharge.

The patient was referred to our hospital due to persistent high fever and hemoptysis. The patient was conscious, cooperative, and poor in orientation. In the respiratory system examination, breathing sounds were reduced in both lungs and there were inspiratory rales in the right middle and lower left lungs.

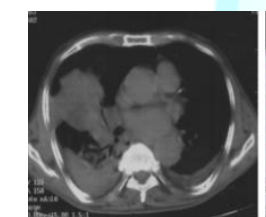

$2 \mathrm{c}$

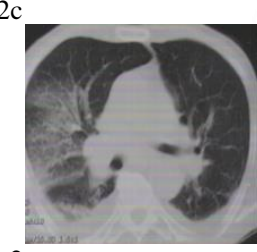

$2 \mathrm{e}$

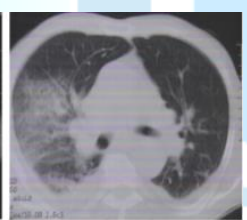

$2 \mathrm{~d}$

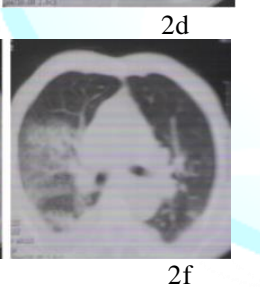

Figure 2c-2f: Chest computed tomography revealed parenchymal consolidation with air bronchogram in the right lower lobe, acinar consolidated areas in the middle lobe and bilateral pleural effusion, which is more pronounced on the right.

Bronchoscopy revealed no endobronchial lesion. Mucosa of right middle and lower lobes were with edema. Cytology showed no lipidladen macrophage. Ampicillin-resistant E. coli and C. Albicans were seen as a result of bronchoalveolar lavage. The patients antibiotic treatment was continued with cefuroxime sodium and ciprofloxacin. The thoracentesis performed from the patients pleural effusion was consistent with exudates. Oral antibiotic was initiated on the 7 th day. The patient was discharged on the 14th day of treatment. Verbal consent was obtained from the patient for this case presentation.

\section{Discussion}

The exogenous lipoid pneumonia is caused by aspiration or inhalation of mineral oils, animal oils or petroleum products or lipoid pneumonia can be endogenous commonly following post obstruction either due to lung cancers, bronchiolitis or lung necrosis [3]. One of the our patients who presented with lipoid exogenous pneumonia was diesel oil aspiration and the other one was admitted to ER as a result of gas oil aspiration.

In the literature, case reports related to aspiration-induced lipoid pneumonias were reported in childhood. However, lipoid pneumonia due to oil aspiration can also be seen in elderly patients. Our patient who was exposed to gas oil aspiration was 72-years-old man. The clinical symptoms of lipoid pneumonia are nonspecific and may vary due to patients age, duration of oil intake; the amount and quality of oil aspirated [4]. In the literature, time of exposure, amounts of oils and quality of material vary according to case reports. Elder patients are more asymptomatic than early ages and also lipoid pneumonias are commonly chronic and progressive for geriatric patients [4]. Because of this reason, lipoid pneumonias are usually discovered by as an autopsy finding [4]. Both of our patients presented to the emergency department with severe clinical presentation.

The diagnosis of exogenous lipoid pneumonia is based on a history of exposure to oils and clinical findings, radiological findings, presence of lipid-laden macrophages on sputum or bronchoalveolar lavage [4]. In despite of, chest radiographs may be unremarkable in patients with exogenous lipoid pneumonia; most of them show abnormalities [5]. Homogenous dense consolidation with air bronchograms, diffuse or focal and unilateral or bilateral involvement, cavitation, nodules and masses resulting from inflammation or fibrosis, atelectasis and pleural effusions may seen on chest radiographs of these patients [5]. Chest computed tomography and magnetic resonance imaging may detect fats within pulmonary tissues [5]. However, that none of these clinical and radiological findings alone is diagnostic for exogenous lipoid pneumonia.

Treatment modalities of lipoid pneumonia are poorly defined. Treatment strategies may include whole lung lavage, supportive care, systemic corticosteroids and thoracoscopy with surgical debridemen [6]. Antibiotics treatment may be given for complicated patients. Lipoid pneumonia is an uncommon disease encountered in all age groups. Physician should enquire about oil intake in all patients with persistent cough and chest symptoms. Treatment involves removal of the offending agent and supportive care.

\section{References}

1. Hadda V and Khilnani GC. Lipoid pneumonia: An overview (2010) Expert Rev Respir Med 4: 799-807. https://doi.org/10.1586/ers.10.74

2. Betancourt SL, Martinez-Jimenez S, Rossi SE, Troug MT, Carrillo J, et al. Lipoid pneumonia: Spectrum of clinical and radiologic manifestations (2010) Cardiopulmonary Imaging 194: 103-105. https://doi.org/10.2214/AJR.09.3040

3. Sharma A, Ohri S, Bambery $\mathrm{P}$ and Singh S. Idiopathic endogenous lipoid pneumonia (2006) Indian J Chest Dis Allied Sci 48: 143-145. https://doi.org/10.1016/i.rmed.2010.12.001

4. Marchiori E, Zanetti G, Mano CM and Hochhegger B. Exogenous lipoid pneumonia. Clin radiological manifestations (2011) Respiratory Med 105: 659-66. https://doi.org/10.1016/j.rmed.2010.12.001

5. Banjar H. Lipoid Pneumonia: A review (2003) Bahrain Medical Bulletin 25: 1-6.

6. Shaikh A and Oliveire PJ. Exogenous lipoid pneumonia (Fire-eaters Lung) (2014) American J Med 127: 3-4. https://doi.org/10.1016/j.amjmed.2013.10.008 